\title{
Research Article \\ Examples of Stability of Tensor Products in Positive Characteristic
}

\author{
A. S. Argáez \\ Universidad Autónoma, "Benito Juárez" de Oaxaca, Mexico \\ Correspondence should be addressed to A. S. Argáez, as_argaez@yahoo.com.mx
}

Received 5 June 2011; Accepted 3 July 2011

Academic Editor: V. Futorny

Copyright (C 2011 A. S. Argáez. This is an open access article distributed under the Creative Commons Attribution License, which permits unrestricted use, distribution, and reproduction in any medium, provided the original work is properly cited.

Let $X$ be projective smooth variety over an algebraically closed field $k$ and let $\mathcal{\varepsilon}, \mathcal{F}$ be $\mu$-semistable locally free sheaves on $X$. When the base field is $\mathbb{C}$, using transcendental methods, one can prove that the tensor product $\mathcal{E} \otimes \mathcal{F}$ is always a $\mu$-semistable sheaf. However, this theorem is no longer true over positive characteristic; for an analogous theorem one needs the hypothesis of strong $\mu$ semistability; nevertheless, this hypothesis is not a necessary condition. The objective of this paper is to construct, without the strongly $\mu$-semistability hypothesis, a family of locally free sheaves with $\mu$-stable tensor product.

\section{Introduction}

When the base field is $\mathbb{C}$, the Kobayashi-Hitchin correspondence ensures that a vector bundle on a complex projective variety is polystable if and only if it admits a Hermitian-Einstein metric with respect to the Kähler metric induced by $\mathcal{O}_{X}(1)$ (see [1] for curves and [2] for complex compact varieties). In this way, one can prove that the tensor product of HermitianEinstein bundles is again Hermitian-Einstein, therefore polystable, and the same is true for symmetric and exterior products. However, in positive characteristic, this is false; in [3], Gieseker proved the existence of stable vector bundles on curves with nonsemistable symmetric products. When these bundles are of degree zero, the nonsemistability of the symmetric product imply the nonsemistability of the tensor product. One way to solve this problem is to introduce the concept of strong $\mu$-semistability. Let $F: X \rightarrow X$ be a total Frobenius morphism of $X$; we say that $\varepsilon$ is strongly $\mu$-semistable if for all $m \geq 0$ the pullback $\left(F^{m}\right)^{*} \mathcal{\varepsilon}$ is $\mu$-semistable with respect to the induced polarization $\left(F^{m}\right)^{*} \mathcal{O}_{X}(1)$. Under these assumptions, the tensor product of strongly $\mu$-semistable bundles is again strongly $\mu$ semistable (see [4, Section 7] for curves and [5] for general case). However, in general, there are no conditions to ensure the $\mu$-semistability of a tensor product of $\mu$-semistable bundles (at least not known to the author). 
The aim of this work is the construction of examples, in any characteristic, of families of $\mu$-stable bundles with $\mu$-stable tensor products, this without the assumption of strong $\mu$ stability. The key result is Proposition 3.1, which shows that, if $\pi: Y \rightarrow X$ is étale and Galois with g.c.d. $(|G|$, char $(k))=1$, then $\mp$ is $\mu$-semistable ( $\mu$-polystable) if and only if $\pi^{*} \mp$ is $\mu$-semistable ( $\mu$-polystable, resp.). We remark that, in positive characteristic, this result is false for an arbitrary finite morphism. Under these hypotheses, if $\mathcal{L}$ is a line bundle on $Y$, in Corollary 3.2 we show that $\mathcal{F} \otimes \pi_{*} \mathcal{\perp}$ is $\mu$-(semi)polystable if $\mathcal{F}$ is $\mu$-(semi)stable. If in addition g.c.d.(deg $\mathscr{F}, r k(\mathcal{F}))=1=(|G|, r k(\mathcal{F}))$ and $g^{*} \mathcal{\perp} \neq \mathcal{\perp}$ for all $g \in G$, then in Corollary 3.5 it is proved that $\mathscr{F} \otimes \pi_{*} \mathcal{\perp}$ is $\mu$-stable. The other examples that are constructed come from the Ginvariant decomposition of $\pi_{*} \mathcal{O}_{Y}$. Now, the general outline of this construction is as follows. In Section 1, we observe that $\pi_{*} \mathcal{O}_{Y}$ has a natural G-invariant decomposition:

$$
\pi_{*} \mathcal{O}_{Y}=\bigoplus_{V \in \mathcal{O}} V \otimes_{k} \varepsilon_{V}
$$

where $\mathcal{J}$ is the set of irreducible representations of $G$ over $k$ and each $\varepsilon_{V}$ is a locally free sheaf of rank $\operatorname{dim} V$. Also, we have the relations

$$
\mathcal{E}_{V_{1}} \otimes \mathcal{E}_{V_{2}} \simeq \bigoplus_{T \in \mathcal{S}} \mathcal{E}_{T}^{\oplus n_{T}}, \quad \wedge^{\varsigma} \mathcal{E}_{V} \simeq \bigoplus_{W \in S} \mathcal{E}_{W}^{\oplus n_{W}}
$$

where $V \otimes W \simeq \bigoplus_{T \in O} T^{\oplus n_{T}}$ and $\wedge^{s} V=\bigoplus_{W \in O} W^{\oplus n_{W}}$. In Section 2, we show that $\varepsilon_{V}$ is $\mu$-H-stable for any polarization on $X$ and also that $\mathcal{F} \otimes \mathcal{E}_{V}$ is $\mu$-H-polystable $(\mu-H$-semistable) whenever $\mp$ is $\mu$-H-stable $\left(\mu\right.$-H-semistable, resp.). Furthermore, let $\mathcal{M}_{X}(r, d)$ be the moduli space parametrizing $\mu$ - $H$-stable sheaves of rank $r$ and degree $d$, if g.c.d. $(d, r)=1=$ g.c.d. $(|G|, r)$, we proved in Theorem 3.12 that, for any irreducible representation $V$ of $G$ and $\mp \in \mathcal{M}_{X}(r, d)$, $\mathcal{F} \otimes \mathcal{E}_{V}$ is $\mu$-H-stable and the natural morphism $\mathcal{M}_{X}(r, d) \rightarrow \mathcal{M}_{X}(r \cdot \operatorname{dim} V, d \cdot \operatorname{dim} V)$ given by $\mathcal{F} \mapsto \mathcal{F} \otimes \mathcal{E}_{V}$ is injective.

In Section 3, we proved that, if g.c.d. $(d,|G|)=1$, then, for all $r>0, G$ acts without fixed points on $\mathcal{M}_{Y}(r, d)$; in particular the morphisms $\mathcal{M}_{Y}(r, d) \rightarrow \mathcal{M}_{Y}(r, d) / G$ are étale Galois covers, thus we have nontrivial examples for Section 2. Finally, when $X$ is an abelian variety and $\pi: Y \rightarrow X$ is an isogeny of degree $r$, Proposition 4.5 shows that, if g.c.d $(r, d)=1$, then each irreducible component of $\operatorname{Pic}^{d}(Y) / G$ is an irreducible component of $\mathcal{M}_{X}(r, d)$, where $G=\operatorname{Ker}(\pi)$. Section 5 is devoted to prove Proposition 3.1.

\subsection{Notations and Conventions}

Throughout the paper, $k$ denotes an algebraically closed field and $G$ a finite group satisfying that g.c.d. $(|G|, \operatorname{char}(k))=1$. Also, $X$ denotes a smooth projective variety over $k$ with a fixed polarization, that is, with a fixed ample line bundle $\mathcal{O}_{X}(1)$, and we denote by $H$ any divisor in the linear system $\left|\mathcal{O}_{X}(1)\right|$.

We denote by $k[G]$ the group algebra of $G$ with coefficients in $k$. Also, if $T_{1}$ and $T_{2}$ are representations of $G$ over $k$ then we denote by $\left\langle T_{1}, T_{2}\right\rangle$ the $\operatorname{dim} \operatorname{Hom}_{k}\left(T_{1}, T_{2}\right)^{G}=$ $\operatorname{dim} \operatorname{Hom}_{k[G]}\left(T_{1}, T_{2}\right)$. We will identify vector bundles on $X$ with locally free $\mathcal{O}_{X}$-modules. 


\section{2. Étale Covers}

Let $\pi: Y \rightarrow X$ be an étale Galois cover. Now, we recall that a locally free sheaf $\mathcal{F}$ on $Y$ is a $G$-sheaf (see [6, page 69]) if $G$ acts on $\mathcal{F}$ in a way compatible with the action on $Y$. Since $\pi$ is étale, it is flat; hence, we have that $\pi_{*} \mathcal{F}$ is a locally free $\mathcal{O}_{X}$-module with an action of $G$. From this, we have defined a natural morphism of $k$-algebras $k[G] \rightarrow \operatorname{End}_{\mathcal{O}_{X}}\left(\pi_{*} \mathscr{F}\right)$. Now, as we suppose by hypothesis that g.c.d. $(\operatorname{char}(k),|G|)=1$, Maschke's Theorem guarantees that $k[G]$ is a semisimple $k$-algebra of finite dimension $|G|$ over $k$. We denote by $\mathcal{J}=\left\{V_{0}, \ldots, V_{r}\right\}$ the set of irreducible representations of $G$ over $k$, that is, the set of irreducible $k[G]$-modules, and by $\left\{e_{V}\right\}_{V \in \mathcal{O}}$ the set of corresponding idempotents. Thus, we have that

$$
\pi_{*} \mathcal{F}=\bigoplus_{V \in S} e_{V}\left(\pi_{*} \mathcal{F}\right)
$$

On the other hand, by Theorem 1(B) in [6, page 111], there is a locally free sheaf $\mathscr{t}$ on $X$ such that $\mathcal{F} \simeq \pi^{*} \mathscr{d}$, so we have natural $G$-invariant isomorphisms $\pi_{*} \mathcal{F} \simeq \pi_{*} \pi^{*} \mathscr{\ell} \simeq$ $\mathscr{H} \otimes \pi_{*} \mathcal{O}_{Y} \simeq \bigoplus_{V \in O} \mathscr{H} \otimes e_{V}\left(\pi_{*} \mathcal{O}_{Y}\right)$; therefore, to understand the G-structure of $\pi_{*} \mathcal{F}$ it suffices to do it for $\pi_{*} \mathcal{O}_{Y}$. Thus, we have the next.

Proposition 2.1. Let $X$ be a smooth projective variety over an algebraically closed field $k$ and $\pi$ : $Y \rightarrow X$ and étale Galois cover with Galois group $G$. Let $V \in \supset$ and define $\mathcal{E}_{V}$ as the $\mathcal{O}_{X}$-module $\pi_{*}\left(V^{*} \otimes_{k} \mathcal{O}_{Y}\right)^{G}=e_{U}\left(\pi_{*}\left(V^{*} \otimes_{k} \mathcal{O}_{Y}\right)\right)$, where $V^{*}$ is the dual representation of $V$ and $U$ is the trivial representation. Then,

(1)

$$
\pi_{*} \mathcal{O}_{Y}=\bigoplus_{V \in \mathcal{S}} V \otimes_{k} \varepsilon_{V}
$$

where $\supset$ is the set of all irreducible representations of $G$ over $k$ and $\operatorname{rank} \varepsilon_{V}=\operatorname{dim}_{k} V$. Also, one has that $\mathcal{\varepsilon}_{V}=e_{U}\left(V^{*} \otimes e_{V}\left(\pi_{*} \mathcal{O}_{Y}\right)\right)$ and $\varepsilon_{V^{*}} \simeq \mathcal{\varepsilon}_{V^{\prime}}^{*}$

(2)

$$
\varepsilon_{V_{i}} \otimes \varepsilon_{V_{j}} \simeq \bigoplus_{l=0}^{r} \mathcal{E}_{V_{l^{\prime}}}^{n_{l}}
$$

where $V_{i} \otimes V_{j}=\bigoplus_{l=0}^{r} V_{l}^{n_{l}}$,

(3) each $\varepsilon_{V}$ is simple (i.e., $\operatorname{End}_{\mathcal{O}_{X}}\left(\mathcal{\varepsilon}_{V}\right) \simeq k$ ) and $\mathcal{\varepsilon}_{V_{i}} \nsucc \mathcal{\varepsilon}_{V_{j}}$ if $i \neq j$,

(4) for any irreducible representation $V$ of $G$ one has that

$$
\wedge^{k} \mathcal{E}_{V}=\bigoplus \mathcal{\varepsilon}_{V_{i}}^{n_{i}}
$$

where $\wedge^{k}(V)=\bigoplus V_{i}^{n_{i}}$. 
Proof. We recall that $k[G] \simeq \bigoplus_{V \in \supset} V \otimes_{k} V^{*}$ as $k[G \times G]$-modules, where actions are given by $g_{1} \times g_{2}\left(\sum \lambda_{g} g\right)=\sum \lambda_{g} g_{1} \cdot g \cdot g_{2}^{-1}$ and $g_{1} \times g_{2}\left(\sum v \otimes \delta\right)=\sum g_{1}(v) \otimes g_{2}(\delta)$, respectively. Thus, we have the following $G$-invariant isomorphisms:

$$
\begin{aligned}
\pi^{*}\left(\bigoplus_{V \in \mathcal{S}} V \otimes_{k} \varepsilon_{V}\right) & =\bigoplus_{V \in \mathcal{S}} V \otimes_{k} \pi^{*}\left(\varepsilon_{V}\right) \simeq\left(\bigoplus_{V \in \mathcal{S}} V \otimes_{k} V^{*}\right) \otimes_{k} \mathcal{O}_{Y} \\
& \simeq k[G] \otimes_{k} \mathcal{O}_{Y} \simeq \bigoplus_{g \in G} g^{*} \mathcal{O}_{Y} \simeq \pi^{*} \pi_{*} \mathcal{O}_{Y}
\end{aligned}
$$

Now, Theorem 1 in [6, page 111] asserts that $\pi^{*}$ defines an equivalence between the category of locally free $\mathcal{O}_{X}$-modules of finite rank and the category of locally free $\mathcal{O}_{Y}$-modules of finite rank with $G$-action. This proves that $\pi_{*} \mathcal{O}_{Y} \simeq \bigoplus_{V \in S} V \otimes_{k} \mathcal{E}_{V}$. In particular, we have that $\mathcal{E}_{V}=$ $e_{U}\left(V^{*} \otimes e_{V}\left(\pi_{*} \mathcal{O}_{Y}\right)\right)$, where $U$ is the trivial $k[G]$-module.

On the other hand, at the generic point $\epsilon$ of $X,\left(\pi_{*} \mathcal{O}_{Y}\right)_{\epsilon}$ is the function field $K_{Y}$ of $Y$ which, by the normal basis theorem, is isomorphic to $K_{X}[G]$ as $K_{X}[G]$-modules, where $K_{X}$ is the function field of $X$. Thus, we have a natural $K_{X}[G]$-isomorphisms:

$$
\left(\pi_{*} \mathcal{O}_{Y}\right)_{\epsilon}=\bigoplus_{V \in \mathcal{S}} V \otimes_{k}\left(\varepsilon_{V}\right)_{\epsilon} \simeq K_{X}[G]=\bigoplus_{V \in \mathcal{O}}\left(V \otimes_{k} K_{X}\right)^{\operatorname{dim} V}
$$

and we conclude that $\varepsilon_{V}$ is a locally free sheaf with rank ( $\left.\operatorname{dim} V\right)$.

For the last part of (1), we need to see that $\left(\mathcal{E}_{V_{i}}\right)^{\vee} \simeq \mathcal{E}_{V_{i}^{\vee}}$, but this is a consequence that the trace morphism $\operatorname{Tr}: \pi_{*} \mathcal{O}_{Y} \rightarrow\left(\pi_{*} \mathcal{O}_{Y}\right)^{\vee}$ is a $G$-invariant isomorphism.

(2) This is immediate from

$$
\begin{aligned}
\pi_{*}\left[\left(V \otimes_{k} W\right) \otimes_{k} \mathcal{O}_{Y}\right]^{G} & =\pi_{*}\left[\bigoplus_{T \in \mathcal{O}}\left(T \otimes_{k} \mathcal{O}_{Y}\right)^{\langle T, V \otimes W\rangle}\right]^{G} \\
& =\bigoplus_{T \in \mathcal{S}} \pi_{*}\left[\left(T \otimes_{k} \mathcal{O}_{Y}\right)^{\langle T, V \otimes W\rangle}\right]^{G}=\bigoplus_{V \in \mathcal{S}} \boldsymbol{\varepsilon}_{T}^{\langle T, V \otimes W\rangle} .
\end{aligned}
$$

(3) We observe that

$$
\operatorname{Hom}_{\mathcal{O}_{X}}\left(\mathcal{E}_{V}, \mathcal{\varepsilon}_{W}\right)=\left[\operatorname{Hom}_{\mathcal{O}_{Y}}\left(V \otimes_{k} \mathcal{O}_{Y}, W \otimes_{k} \mathcal{O}_{Y}\right)\right]^{G}=\operatorname{Hom}_{k[G]}(V, W),
$$

and from Schur's Lemma, $\operatorname{dim}_{k} \operatorname{Hom}_{k[G]}(V, W)$ is 0 if $V \neq W$ and 1 if $V \simeq W$ (see [7, page 181]).

(4) We know that tensor functors commutes with the pull back, so we have that

$$
\begin{aligned}
\pi^{*}\left(\wedge^{k} \mathcal{\varepsilon}_{V}\right) & \simeq \wedge^{k}\left(\pi^{*} \mathcal{\varepsilon}_{V}\right) \simeq \wedge^{k}\left(V^{*} \otimes \mathcal{O}_{Y}\right) \simeq\left(\wedge^{k} V^{*}\right) \otimes \mathcal{O}_{Y} \simeq \bigoplus_{T \in \mathcal{S}}\left(T^{*}\right)^{\left\langle T, \wedge^{k} V\right\rangle} \otimes \mathcal{O}_{Y} \\
& \simeq \bigoplus_{T \in \mathcal{S}}\left(T^{*} \otimes \mathcal{O}_{Y}\right)^{\left\langle T, \wedge^{k} V\right\rangle} \simeq \bigoplus_{T \in \mathcal{O}} \pi^{*}\left(\mathcal{\varepsilon}_{T}^{\left\langle T, \wedge^{k} V\right\rangle}\right) \simeq \pi^{*}\left(\bigoplus_{T \in \mathcal{S}} \mathcal{\varepsilon}_{T}^{\left\langle T, \wedge^{k} V\right\rangle}\right)
\end{aligned}
$$

and, applying Theorem 1(B) in [6, page 111], we get the desired isomorphism. 
Remark 2.2. Note that (4) is valid for each of the Schur functors.

\section{Stable Sheaves}

Let $\mathcal{\varepsilon}$ be a locally free sheaf over $X$ and

$$
m \longmapsto \chi(\mathcal{E} \otimes \mathcal{O}(m))
$$

its Hilbert polynomial, where $X(\varepsilon)=\sum(-1)^{i} h^{i}(X, \varepsilon)$ is the Euler characteristic of $\varepsilon$. It is well known that this polynomial can be written as

$$
P(\mathcal{\varepsilon}, m)=\sum_{i=0}^{d} \alpha_{i}(\mathcal{\varepsilon}) \frac{m^{i}}{i !} .
$$

Define the degree of $\varepsilon$ as

$$
\operatorname{deg}(\mathcal{\varepsilon})=\alpha_{d-1}(\mathcal{\varepsilon})-r k(\mathcal{\varepsilon}) \alpha_{d-1}\left(\mathcal{O}_{X}\right)
$$

and its slope by

$$
\mu(\mathcal{\varepsilon})=\frac{\operatorname{deg}(\mathcal{\varepsilon})}{r k(\mathcal{\varepsilon})}
$$

where $r k(\varepsilon)$ is the rank of $\varepsilon$. Recall that a locally free sheaf is $\mu$-H-stable ( $\mu$-H-semistable) if $\mu(\mathcal{F})<\mu(\mathcal{E})$ for all subsheaf $\mathscr{F}(\mu(\mathcal{F}) \leq \mu(\mathcal{E})$, resp.); also a $\mu-H$-semistable locally free sheaf is said $\mu$-H-polystable if it is a direct sum of $\mu$-H-stable sheaves. Any $\mu$-H-stable sheaf is simple, that is, End $(\mathcal{E})=k$, in particular, a $\mu$ - $H$-polystable sheaf is $\mu-H$-stable if and only if it is a simple sheaf.

The following proposition is proved in [8, pages 62-63] under the assumption of characteristic zero in the base field, but the arguments that surround the proof are valid for any characteristic when we consider only étale covers; however, per clarity we will repeat the proof in Section 5.

Proposition 3.1. Let $X$ be a complete variety over $k$ and $\pi: Y \rightarrow X$ be an étale Galois cover, with Galois group G. Let $\varepsilon$ be a locally free sheaf on X. Then, $\varepsilon$ is $\mu$-H-polystable if and only if $\pi^{*} \varepsilon$ is $\mu$ - $\pi^{*} H$-polystable.

Corollary 3.2. Let $\mathcal{L}$ be a line bundle on $Y$; then; for all $\mu$-H-(semi)stable bundle $\mathcal{F}$ on $X, \mathcal{F} \otimes \pi_{*} \mathcal{\perp}$ is $\mu$-H-(semi)polystable bundle.

Proof. We have that the cover is étale, thus $\pi^{*}\left(\mathcal{F} \otimes \pi_{*} \mathcal{L}\right) \simeq \pi^{*}\left(\pi_{*} \pi^{*} \mathcal{\Psi} \otimes \mathcal{L}\right) \simeq \bigoplus_{g \in G} g^{*}\left(\pi^{*} \mathcal{F} \otimes \mathcal{L}\right) \simeq$ $\bigoplus_{g \in G} \pi^{*} \mathcal{F} \otimes g^{*} \mathcal{L}$. Then, by Proposition 3.1, $\mathcal{F} \otimes \pi_{*} \mathcal{L}$ is $\mu$ - $H$-(semi) polystable because $\pi^{*} \mathcal{F} \otimes g^{*} \mathcal{L}$ is $\mu-\pi^{*} H$-(semi)polystable.

Corollary 3.3. Let $\mathcal{G}$ be a locally free $\mu-\pi^{*} H$-stable $\mathcal{O}_{Y}$-module. Then, $\pi_{*} \mathcal{G}$ is $\mu$-H-polystable, and is $\mu$-H-stable if and only if $\mathcal{G} \neq g^{*} \mathcal{G}$ for all $g \neq \mathrm{Id}$. 
Proof. We have that the cover is étale, thus $\pi^{*} \pi_{*} \mathcal{G} \simeq \bigoplus_{g \in G} g^{*} \mathcal{G}$, so $\pi_{*} \mathcal{G}$ is $\mu$-H-polystable because $g^{*} \mathcal{G} \simeq \mathcal{G}$ is $\mu$ - $\pi^{*} H$-stable. On the other hand,

$$
\operatorname{dim} \operatorname{Hom}\left(\pi_{*} \mathcal{G}, \pi_{*} \mathcal{G}\right)=h^{0}\left(Y,\left[\bigoplus_{g \in G} g^{*} \mathcal{G}\right] \otimes \mathcal{G}^{*}\right)=\sum_{g \in G} h^{0}\left(Y, g^{*} \mathcal{G} \otimes \mathcal{G}\right)
$$

so $\pi_{*} \mathcal{G}$ is simple if and only if $g^{*} \mathcal{G} \neq \mathcal{G}$ for all $g \neq 1 d_{Y}$.

Now, we recall the following lemma of the theory of semistable bundles.

Lemma 3.4. Let $\mathcal{\varepsilon}$ be a $\mu$-H-semistable sheaf; if g.c.d $(\operatorname{deg}(\varepsilon), r k(\varepsilon))=1$, then $\mathcal{\varepsilon}$ is $\mu$-H-stable.

Proof. If $\varepsilon$ is not $\mu-H$-stable, then there exists a subsheaf $\mathcal{F}$ such that $\mu(F)=\mu(\varepsilon)$ and $r k(\mathcal{F})<r k(\mathcal{E})$; thus, we have $\operatorname{deg}(\mathscr{F}) r k(\mathcal{E})=\operatorname{deg}(\mathcal{E}) r k(\mathcal{F})$ which contradicts the assumption g.c.d. $(\operatorname{deg}(\mathcal{\varepsilon}), r k(\mathcal{\varepsilon}))=1$.

Corollary 3.5. Let $d, r$ be integers such that, $r>0$, g.c.d. $(d, r)=1=$ g.c.d. $(|G|, r)$, and suppose that $\mathcal{L}$ is a line bundle on $Y$ such that $\pi_{*} \mathcal{\perp}$ is $\mu$-H-stable; then, for all $\mu$-H-stable bundle $\mathcal{F}$ on $\mathrm{X}$ of degree $d$ and $\operatorname{rank} r$, one has that $\mathcal{F} \otimes \pi_{*} \mathcal{\perp}$ is $\mu$-H-stable bundle.

Proof. From Proposition 3.1, we have that $\pi^{*} \mp$ is $\mu-\pi^{*} H$-polystable with degree $d|G|$ and rank $r$, and from the fact that g.c.d. $(d|G|, r)=1$ and Lemma 3.4, we have that it is $\mu$ - $\pi^{*} H$-stable. Now, by Corollary 3.3, $\pi_{*} \mathcal{L}$ is $\mu$-H-stable if and only if $g^{*} \mathcal{L} \neq \mathcal{L}$ for all $g \in G$; thus, $\pi^{*} \mathcal{F} \otimes$ $g^{*} \mathcal{L} \neq \pi^{*} \mathcal{F} \otimes \mathcal{L}$ for all $g \in G$, then $\pi_{*}\left(\pi^{*} \mathcal{F} \otimes \mathcal{L}\right) \simeq \mathscr{F} \otimes \pi_{*} \mathcal{L}$ is $\mu$-H-stable.

Theorem 3.6. Let $X$ be a smooth projective variety over an algebraically closed field $k$ and let $\pi$ : $Y \rightarrow X$ be an étale Galois cover with Galois group $G$ and

$$
\pi_{*} \mathcal{O}_{Y}=\bigoplus_{V \in \mathcal{O}} V \otimes_{k} \varepsilon_{V}
$$

the isotypical decomposition of $\pi_{*} \mathcal{O}_{Y}$. Then, each $\varepsilon_{V}$ is $\mu$-H-stable with respect to any ample line bundle $\mathcal{O}_{X}(1)$.

Proof. As $\pi: Y \rightarrow X$ is étale, we have that $\pi^{*} \pi_{*} \mathcal{O}_{Y} \simeq \mathcal{O}_{Y}^{\oplus|G|}$, and from Proposition 3.1, it follows that $\pi_{*} \mathcal{O}_{Y}$ is $\mu$-H-polystable; now, we just have to see that each $\mathcal{E}_{V}$ is simple, but this is consequence of Proposition 2.1, Part (3).

Corollary 3.7. Let $\mathcal{F}$ be a $\mu$-H-semistable vector bundle on $X$, then $\varepsilon_{V} \otimes \mathcal{F}$ is $\mu$-H-semistable for all $\varepsilon_{V}$. Moreover, if $\mathcal{F}$ is $\mu$-H-stable, then $\varepsilon_{V} \otimes \mathcal{F}$ is $\mu$-H-polystable.

Proof. We have that $\pi^{*} \pi_{*} \pi^{*} \mathcal{F} \simeq \bigoplus_{g \in G} \pi^{*} \mathcal{F}$, so, by Lemma 5.3 of Section $5, \pi_{*} \pi^{*} \mathcal{F}$ is $\mu-H$ semistable; now, $\pi_{*} \pi^{*} \mathcal{F} \simeq \mathcal{F} \otimes \pi_{*} \mathcal{O}_{Y} \simeq \bigoplus_{V \in S} V \otimes \mathcal{E}_{V} \otimes \mathcal{F}$, then $\mathcal{E}_{V} \otimes \mathcal{F}$ is $\mu$-H-semistable for all $V$. Applying Proposition 3.1 we get the second assertion by an analogous argument.

Let $\mathcal{M}_{H}(r, d)\left(\overline{\mathcal{M}_{H}(r, d)}\right)$ be the moduli space parametrizing locally free $\mu$ - $H$-stable sheaves ( $\mu$-H-polystables, resp.), of rank $k$ and degree zero over $k$ over $X$. 
Corollary 3.8. Let X be a smooth projective variety over an algebraically closed field $k$. Suppose that $X$ admits an étale Galois cover with Galois group $G$. Then, for all irreducible representation $V$ of $G$ over $k$ and any polarization $H$, one has that $\mathcal{M}_{H}(\operatorname{dim} V, 0)$ is non empty.

Now, on characteristic zero, we have that exterior products of the standard representation of symmetric groups are irreducible (see [9, page 31]); thus, we have the next.

Corollary 3.9. Let $X$ be a smooth projective variety over $k$, $\operatorname{char}(k)=0$, and let $H$ be any polarization. Suppose that the variety admits a Galois cover with Galois group, the symmetric group on d letters $\mathfrak{S}_{d}$. Then, there exists a nonempty open set $U \subset \mathcal{M}_{H}(d-1,0)$ such that $\bigwedge^{k} \mathcal{E}$ is $\mu$-H-stable for all $\varepsilon \in U$.

Proof. As the characteristic is zero, the wedge product of $\mu$-H-stables sheaves is $\mu-H$ polystable, in particular $\mu$ - $H$-semistable. So, we have defined a morphism:

$$
\mathscr{M}_{H}(d-1,0) \longrightarrow \overline{\mathcal{M}_{H}\left(\left(\begin{array}{c}
d-1 \\
k
\end{array}\right), 0\right)}
$$

given by $\boldsymbol{\varepsilon} \mapsto \bigwedge^{k} \mathcal{\varepsilon}$. As the variety $\mathcal{M}_{H}\left(\left(\begin{array}{c}d-1 \\ k\end{array}\right), 0\right)$ is an open set of $\overline{\mathcal{M}_{H}\left(\left(\begin{array}{c}d-1 \\ k\end{array}\right), 0\right)}$, we only need to see that there is an $\varepsilon \in \mathcal{M}_{H}(d-1,0)$ such that $\Lambda^{k} \mathcal{\varepsilon}$ is $\mu$ - $H$-stable.

By hypothesis, we assume the existence of an étale Galois cover with Galois group, the symmetric group on $d$ letters $\mathfrak{S}_{d}$; let $V$ be the standard representation of $\mathfrak{S}_{d}$ and $\mathcal{E}_{V}$ the corresponding sheaf obtained in Theorem 3.6, and recalling that in characteristic zero the exterior algebra of the standard representation $\Lambda^{k} V$ is irreducible for all $k$, we have, from part (4) of Proposition 2.1, that $\bigwedge^{k} \mathcal{\varepsilon}_{V}=\mathcal{\varepsilon}_{\Lambda^{k} V}$ is $\mu$-H-stable.

In particular we have the following.

Corollary 3.10. Let $X$ be a smooth projective curve of genus $g>1$ over a field of characteristic zero. Then, $\wedge^{k} \mathcal{\varepsilon}$ is $\mu$-H-stable for the generic vector bundle $\mathcal{\varepsilon}$ of degree zero and rank $d$.

Proof. In this case, the moduli spaces $\boldsymbol{M}_{H}(d, 0)$ and $\overline{\mathcal{M}_{H}\left(\left(\begin{array}{l}d \\ k\end{array}\right), 0\right)}$ are irreducible, so we only need to prove the existence of a Galois cover with Galois group $\mathfrak{S}_{d+1}$ and, by the Lefschetz Principle (see [10]), it suffices to do it for $k=\mathbb{C}$. Let $\pi_{1}\left(X, x_{0}\right)=$ $\left\langle\alpha_{1}, \beta_{1}, \ldots, \alpha_{g}, \beta_{g} \mid \Pi\left[\alpha_{i} \beta_{i} \alpha_{i}^{-1} \beta_{i}^{-1}\right]=1\right\rangle$ be the fundamental group of $X$, with base point $x_{0}$; on the other hand, the symmetric group $\mathfrak{S}_{d+1}$ is generated by one transposition and one cycle of length $d+1$, so we can define a surjective morphism $\pi_{1}\left(X, x_{0}\right) \rightarrow \mathfrak{S}_{d+1}$, for example, the one given by $\alpha_{1} \mapsto(1,2), \alpha_{2} \mapsto(1, \ldots, d+1), \alpha_{i} \mapsto 1$ if $i>2$ and $\beta_{1} \mapsto(1,2)$, $\beta_{2} \mapsto(1, \ldots, d+1)^{-1}=(d+1, d, d-1, \ldots, 2,1), \beta_{i} \mapsto 1$ if $i>2$.

Proposition 3.11. Let $X$ be projective smooth variety over an algebraically closed field $k$ and let $\pi: Y \rightarrow X$ be an étale Galois cover with Galois group $G$, and set $\pi_{*} \mathcal{O}_{Y}=\bigoplus_{V \in I} V \otimes_{k} \mathcal{\varepsilon}_{V}$. Let $\mathcal{F}$ be a $\mu$-H-stable vector bundle. Then, the following statements are equivalent:

(1) $\pi^{*} \mp$ is $\mu-\pi^{*} H$-stable;

(2) $\operatorname{Hom}\left(\mathcal{F}, \mathcal{F} \otimes \mathcal{E}_{V}\right)=0$ for all $V \neq V_{0}$;

(3) $\mathcal{F} \otimes \mathcal{E}_{V}$ is $\mu$-H-stable for all $V$ and $\mathcal{F} \otimes \mathcal{E}_{V} \neq \mathcal{F} \otimes \mathcal{E}_{W}$ for $V \neq W$. 
Proof. (1) $\Leftrightarrow(2)$ Since $\mathcal{F}$ is $\mu$ - $H$-stable, from Proposition 3.1, we have that $\pi^{*} \mathcal{F}$ is $\mu-\pi^{*} H$ polystable; on the other hand,

$$
\begin{aligned}
\operatorname{Hom}\left(\pi^{*} \mathcal{F}, \pi^{*} \mathscr{F}\right) & =H^{0}\left(Y, \pi^{*} \mathcal{F}^{\vee} \otimes \pi^{*} \mathcal{F}\right)=H^{0}\left(X, \pi_{*}\left[\pi^{*} \mathcal{F}^{\vee} \otimes \pi^{*} \mathscr{F}\right]\right) \\
& =H^{0}\left(X, \mathcal{F}^{\vee} \otimes \pi_{*} \pi^{*} \mathcal{F}\right)=\operatorname{Hom}\left(\mathcal{F}, \pi_{*} \pi^{*} \mathcal{F}\right)=\operatorname{Hom}\left(\mathcal{F}, \mathcal{F} \otimes \pi_{*} \mathcal{O}_{Y}\right) \\
& =\operatorname{Hom}\left(\mathcal{F}, \mathcal{F} \otimes\left[\bigoplus_{V \in I} V \otimes_{k} \varepsilon_{V}\right]\right)=\bigoplus_{V \in I} V \otimes_{k} \operatorname{Hom}\left(\mathcal{F}, \varepsilon_{V} \otimes \mathcal{F}\right) .
\end{aligned}
$$

Thus, $\pi^{*} \mathcal{F}$ is a simple vector bundle if and only if $\operatorname{Hom}\left(\mathcal{F}, \boldsymbol{\varepsilon}_{V} \otimes \mathcal{F}\right)=0$ for all $V$ different from the trivial representation $V_{0}$.

(1) $\Leftrightarrow$ (3) Notice that $\pi^{*} \pi_{*} \pi^{*} \mathcal{F} \simeq \bigoplus g^{*} \pi^{*} \mathcal{F} \simeq \pi^{*} \mathcal{F}^{\oplus|G|}$ is $\mu$ - $\pi^{*} H$-polystable; thus, $\pi_{*} \pi^{*} \mathcal{F} \simeq \mathcal{F} \otimes \pi_{*} \mathcal{O}_{Y}$ and $\mathcal{F} \otimes \mathcal{E}_{V}$ are $\mu$-H-polystable. Also, we have, from Proposition 3.1, that $\pi^{*} \mathcal{F}$ is $\mu-\pi^{*} H$-polystable; then $\pi^{*} \mathcal{F}$ is simple, and then, $\mu-\pi^{*} H$-stable if and only if

$$
\operatorname{dim} \operatorname{Hom}\left(\pi^{*} \mathcal{F},\left(\pi^{*} \mathscr{F}\right)^{\oplus|G|}\right)=|G|
$$

On the other hand, we have the following relations:

$$
\begin{aligned}
& \operatorname{Hom}\left(\pi^{*} \mathcal{F},\left(\pi^{*} \mathcal{F}\right)^{\oplus|G|}\right)=\operatorname{Hom}\left(\pi^{*} \mathcal{F}, \bigoplus_{g \in G} g^{*} \pi^{*} \mathcal{F}\right) \\
& =\operatorname{Hom}\left(\pi^{*} \mathcal{F}, \pi^{*} \pi_{*} \pi^{*} \mathscr{F}\right) \\
& =H^{0}\left(Y, \pi^{*} \mathcal{F}^{\vee} \otimes \pi^{*} \pi_{*} \pi^{*} \mathcal{F}\right) \\
& =H^{0}\left(X, \pi_{*} \pi^{*} \mathcal{F}^{\vee} \otimes \pi_{*} \pi^{*} \mathcal{F}\right) \\
& =\operatorname{Hom}\left(\pi_{*} \pi^{*} \mathcal{F}, \pi_{*} \pi^{*} \mathcal{F}\right) \\
& =\operatorname{Hom}\left(\mathfrak{F} \otimes \mathcal{O}_{Y}, \mathfrak{F} \otimes \mathcal{O}_{Y}\right) \\
& =\operatorname{Hom}\left(\mathcal{F} \otimes\left[\bigoplus_{V \in I} V \otimes_{k} \varepsilon_{V}\right], \mathcal{F} \otimes\left[\bigoplus_{V \in I} V \otimes_{k} \varepsilon_{V}\right]\right) \\
& =\bigoplus_{V, W \in I} \operatorname{Hom}\left(V \otimes_{k} \mathcal{F} \otimes \mathcal{E}_{V}, W \otimes_{k} \mathcal{F} \otimes \mathcal{E}_{W}\right) \\
& \simeq \bigoplus_{V, W \in I} \operatorname{Hom}\left(\mathcal{F} \otimes \mathcal{E}_{V}, \mathcal{F} \otimes \mathcal{E}_{W}\right)^{(\operatorname{dim} V) \times(\operatorname{dim} W)} \\
& \simeq\left[\bigoplus_{V \in I} \operatorname{Hom}\left(\mathcal{F} \otimes \mathcal{E}_{V}, \mathcal{F} \otimes \mathcal{E}_{V}\right)^{(\operatorname{dim} V)^{2}}\right] \\
& \bigoplus\left[\bigoplus_{V \neq W} \operatorname{Hom}\left(\mathcal{F} \otimes \mathcal{\varepsilon}_{V}, \mathcal{F} \otimes \mathcal{\varepsilon}_{W}\right)^{(\operatorname{dim} V) \times(\operatorname{dim} W)}\right] .
\end{aligned}
$$


Now, $|G|=\sum_{V \in I}(\operatorname{dim} V)^{2}$ and, from previous relationships, we have that equality (3.9) is possible if and only if $\operatorname{dimHom}\left(\mathcal{F} \otimes \mathcal{E}_{V}, \mathcal{F} \otimes \mathcal{E}_{W}\right)$ is zero if $V \neq W$ and $\mathscr{F} \otimes \mathcal{E}$ are simple for all $V$. This tested the statement.

Theorem 3.12. Let $d, r$ be integers such that $r>0$ and g.c.d. $(d, r)=1=$ g.c.d. $(|G|, r)$. Then, for every irreducible representation $V$ of $G$ and $\mathcal{F} \in \mathcal{M}_{X}(r, d)$ one has that $\mathcal{F} \otimes \boldsymbol{\varepsilon}_{V}$ is $\mu$-Hstable. In addition, the natural induced morphism $\mathcal{M}_{X}(r, d) \rightarrow \mathcal{M}_{X}(r \cdot \operatorname{dim} V, d \cdot \operatorname{dim} V) \subset$ $\overline{\mathcal{M}_{X}(r \cdot \operatorname{dim} V, d \cdot \operatorname{dim} V)}$ is injective.

Proof. From Proposition 3.1, we have that $\pi^{*} \mp$ is $\mu-\pi^{*} H$-polystable with degree $d|G|$ and rank $r$, and from the fact that g.c.d. $(d|G|, r)=1$ and Lemma 3.4, we have that it is $\mu$ $\pi^{*} H$-stable. Thus, applying Proposition 3.11, we have that $\mathcal{F} \otimes \mathcal{E}_{V}$ is $\mu$-H-stable for any representation $V$.

Let us now prove the injectivity of the morphism $\mathcal{M}_{X}(r, d) \rightarrow \mathcal{M}_{X}(r \cdot \operatorname{dim} V, d \cdot \operatorname{dim} V)$.

Let $\mathcal{F}_{1}, \mathcal{F}_{2} \in \mathcal{M}_{X}(r, d)$ and suppose that $\mathcal{F}_{1} \otimes \mathcal{E}_{V} \simeq \mathcal{F}_{2} \otimes \mathcal{E}_{V} ;$ notice that this isomorphism implies that $\operatorname{det}\left(\mathscr{F}_{1}\right)=\operatorname{det}\left(\mathscr{F}_{2}\right)$. From the $\mu$-H-stability of $\mathscr{F}_{i} \otimes \mathcal{E}_{V}$, we have that these vector bundles are simple, so that $1=\operatorname{dim} \operatorname{Hom}\left(\mathscr{F}_{1} \otimes \mathcal{E}_{V}, \mathcal{F}_{2} \otimes \mathcal{E}_{V}\right)=\operatorname{dim} \operatorname{Hom}\left(\mathscr{f}_{1}, \mathcal{F}_{2} \otimes \mathcal{E}_{V} \otimes \mathcal{E}_{V}^{*}\right)$.

On the other hand, from Proposition 2.1, we have that $\mathcal{E}_{V}^{*}=\mathcal{E}_{V^{*}}$ and $\varepsilon_{V} \otimes \varepsilon_{V^{*}}=\bigoplus \mathcal{E}_{\mathrm{T}}^{\left\langle T, V \otimes V^{*}\right\rangle}$, so $1=\operatorname{dim} \operatorname{Hom}\left(\mathcal{F}_{1} \otimes \mathcal{\varepsilon}_{V}, \mathcal{F}_{2} \otimes \varepsilon_{V}\right)=\bigoplus_{T \in O} \operatorname{dim} \operatorname{Hom}\left(\mathcal{F}_{1}, \mathcal{F}_{2} \otimes \varepsilon_{T}\right)^{\left\langle T, V \otimes V^{*}\right\rangle}$ and we can deduce the existence of a unique representation $T \in \mathcal{O}$ of dimension 1 such that $\mathcal{F}_{1} \simeq \mathcal{F}_{2} \otimes \varepsilon_{T}$. From here we have that $\operatorname{det}\left(\mathcal{F}_{1}\right) \simeq \operatorname{det}\left(\mathcal{F}_{2}\right) \otimes\left(\boldsymbol{\varepsilon}_{T}\right)^{\otimes r}$, where $r=r k\left(\mathcal{F}_{2}\right)$ and, thus, $\left(\boldsymbol{\varepsilon}_{T}\right)^{\otimes r} \simeq \mathcal{O}_{X}$ and applying part (4) of Proposition 2.1, we obtain that $\boldsymbol{\varepsilon}_{T^{\otimes r}}=\mathcal{O}_{\mathrm{X}}$ and so $T^{\otimes r}$ is the trivial representation. However, from representation theory, the order of the cyclic group generated by the isomorphism class of $T$ should be divided by $|G|$, but by hypothesis g.c.d. $(r,|G|)=1$, then $r=1$ and $T$ is the trivial representation.

\section{Examples of Varieties with Group Action: Actions in the Moduli Space of Stable Bundles}

Again, let $\pi: Y \rightarrow X$ be an étale Galois cover with Galois group $G$, thus the action of $G$ on $Y$ determines an action on the moduli space $\mathcal{M}_{Y}(r, d)\left(\overline{\mathcal{M}_{Y}(r, d)}\right)$ of $\mu$-H-(semi)stables sheaves on $Y$, and from Corollary 3.3, we have a natural morphism $\mathcal{M}_{Y}(r, d) \rightarrow \overline{\mathcal{M}_{X}(r|G|, d)}$ given by $\mathcal{G} \mapsto \pi_{*} \mathcal{G}$, which factorizes by the quotient $\mathcal{M}_{Y}(r, d) / G$. The aim of this section is the study of such morphism.

Proposition 4.1. Suppose that g.c.d $(|G|, d)=1$. Then, for all integer $r>0$, the natural action of $G$ on $\mathcal{M}_{Y}(r, d)$ is without fixed points; in particular, for all $\mathcal{G} \in \mathcal{M}_{Y}(r, d)$, one has that $\pi_{*} \mathcal{G}$ is $\mu$-H-stable.

Proof. Let $\operatorname{Pic}_{\mathcal{O}_{Y}(1)}^{d}(Y)$ be the subvariety of the Picard variety Pic $(Y)$ formed by line bundles of degree $d$ with respect to $\mathcal{O}_{Y}(1)=\pi^{*} \mathcal{O}_{X}(1)$. Thus, we have defined the determinant morphism det : $\mathcal{M}_{Y}(r, d) \rightarrow \operatorname{Pic}_{\mathcal{O}_{Y}(1)}^{d}(Y)$ and this satisfies that $\operatorname{det}\left(g^{*}(\mathcal{E})\right)=g^{*}(\operatorname{det}(\mathcal{E}))$, so it suffices to prove the proposition for $\operatorname{Pic}_{\mathcal{O}_{Y}(1)}^{d}(\mathrm{Y})$.

Let $\mathcal{L} \in \operatorname{Pic}_{\mathcal{O}_{Y}(1)}^{d}(Y)$; from Lemma 5.2, we have that $\operatorname{deg}\left(\pi_{*} \mathcal{L}\right)=d$ and $r k\left(\pi_{*} \mathcal{L}\right)=|G|$, so

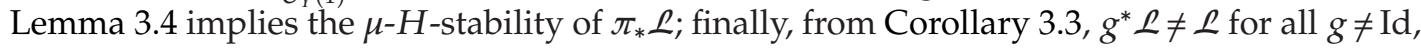
then we conclude that there are no fixed points. The last part of the statement is consequence of Corollary 3.3. 
Denote by $Q_{r, d}^{\pi}$ the quotient variety $\mathcal{M}_{Y}(r, d) / G$ and $\delta: \mathcal{Q}_{r, d}^{\pi} \rightarrow Q_{1, d}^{\pi}$ the induced morphism by det $: \mathcal{M}_{Y}(r, d) \rightarrow \operatorname{Pic}^{d}(Y)=\mathcal{M}_{Y}(1, d)$. The fiber of this map is described in the following.

Corollary 4.2. Let $\mathcal{L} \in \operatorname{Pic}^{d}(Y)$. Then, if $\operatorname{g.c.d}(|G|, d)=1$, then the restriction of the quotient morphism defines an isomorphism $\operatorname{det}^{-1}(\mathcal{L}) \rightarrow \delta^{-1}([\mathcal{L}])$.

Proof. In order to see this, it suffices to show that $g\left(\operatorname{det}^{-1}(\mathcal{L})\right) \cap \operatorname{det}^{-1}(\mathcal{L})=\emptyset$ which is consequence of the proof of the above proposition.

Corollary 4.3. Let $r$ be a positive integer and suppose that g.c.d $(|G|, d)=1$. Then, one has a natural injective morphism $\pi_{*}: Q_{r, d}^{\pi} \rightarrow \mathcal{M}_{X}(r|G|, d) \subset \overline{\mathcal{M}_{X}(r|G|, d)}$.

Consider $Y$ and $X$ curves. If g.c.d. $(r, d)=1$, then we have that $\mathcal{M}_{Y}(r, d)=\overline{\mathcal{M}_{Y}(r, d)}$ and that the moduli space is smooth (see [8, Section 4.5]). So, we have the following.

Corollary 4.4. $\mathcal{Q}_{r, d}^{\pi}$ is a smooth subvariety of $\mathcal{M}_{X}(r|G|, d)$.

In general, there is a natural map $\operatorname{Pic}_{\mathcal{O}_{Y}(1)}^{d}(Y) \rightarrow \mathcal{M}_{X}(|G|, d)$ whose image is naturally isomorphic to the quotient variety $Q_{1, d}^{\pi}$. A special case is for abelian varieties; we note that, by a theorem of Serre-Lang, every étale cover of an abelian variety $X$ has the structure of an abelian variety (see [6, page 167]).

Proposition 4.5. Let $X$ be a polarized abelian variety and $\pi: Y \rightarrow X$ an étale cyclic cover of degree $r$. Let $d$ be an integer such that g.c.d $(r, d)=1$. Then, each irreducible component of $Q_{1, d}^{\pi}$ is a smooth irreducible component of $\mathcal{M}_{X}(r, d)$.

Proof. By previous Corollary 4.4, $Q_{1, d}^{\pi}$ is a smooth subvariety of $\mathcal{M}_{X}(r|G|, d)$ and by Proposition $4.1 g^{*} \mathcal{L} \neq \mathcal{L}$ if $g \neq \mathrm{Id}$. Now, let $\mathcal{L} \in \operatorname{Pic}^{d}(Y)$; thus, $T_{\pi_{*}} \mathcal{L} \mathcal{M}_{X}(r, d)$ is given by $H^{1}\left(X, \pi_{*} \mathcal{\perp} \otimes \pi_{*} \mathcal{L}^{-1}\right) \simeq H^{1}\left(X, \pi_{*}\left(\pi^{*} \pi_{*} L \otimes \mathcal{L}^{-1}\right)\right) \simeq H^{1}\left(Y, \pi^{*} \pi_{*} \mathcal{L} \otimes \mathcal{L}^{-1}\right) \simeq H^{1}\left(Y, \bigoplus_{g \in G} g^{*} \mathcal{\perp} \otimes\right.$ $\left.\mathcal{L}^{-1}\right) \simeq \bigoplus_{g \in G} H^{1}\left(Y, g^{*} \mathcal{L} \otimes \mathcal{L}^{-1}\right)$; now, by the vanishing theorem for abelian varieties in [6, page 76], we have that $h^{1}(L)=0$ for all $\mathcal{L} \in \operatorname{Pic}^{0}(Y), \mathcal{L} \neq \mathcal{O}_{Y}$. Then, $T_{\pi_{*} \perp} \mathcal{M}_{X}(r, d) \simeq H^{1}\left(Y, \mathcal{O}_{Y}\right) \simeq$ $T_{\llcorner} \operatorname{Pic}(Y) \simeq T_{\pi_{*} \perp} Q_{1, d}^{\pi}$.

\section{Proof of Proposition 3.1}

We will need three lemmas; on them, we will be under the assumptions of Proposition 3.1.

Let us recall an equivalent definition of the degree of an $\mathcal{O}_{X}$-module.

Lemma 5.1. For a locally free sheaf $\boldsymbol{\varepsilon}$,

$$
\operatorname{deg}(\varepsilon)=c_{1}(\varepsilon) \cdot H^{d-1}
$$

In particular, if $\mathcal{E}$ and $\mathcal{F}$ are locally free sheaves, then one has

$$
\operatorname{deg}(\mathcal{E} \otimes \mathcal{F})=r k(\mathcal{E}) \operatorname{deg}(\mathcal{F})+r k(\mathcal{F}) \operatorname{deg}(\mathcal{E})
$$


Proof. By Hirzebruch-Riemann-Roch formula, we have

$$
\begin{aligned}
P(\mathcal{E})(m) & =\chi\left(\mathcal{E} \otimes \mathcal{O}_{X}(m)\right)=\operatorname{deg}\left[\operatorname{ch}\left(\mathcal{\varepsilon} \otimes \mathcal{O}_{X}(m)\right) \cdot \operatorname{td}\left(\tau_{X}\right)\right]_{n} \\
& =\sum_{k}\left\{\sum_{i+j=n-k} \operatorname{deg}\left[\operatorname{ch}(\mathcal{\varepsilon})_{i} \cdot \operatorname{td}\left(\tau_{X}\right)_{j} \cdot H^{d-k}\right]\right\} \frac{m^{k}}{k !}
\end{aligned}
$$

where $\operatorname{ch}\left(\mathcal{O}_{X}(m)\right)=\sum_{k}\left((m H)^{k}\right) / k$ ! for some ample divisor $H$; thus,

$$
\begin{aligned}
\alpha_{d-1}(\mathcal{\varepsilon}) & =\operatorname{deg}\left(\operatorname{ch}(\mathcal{\varepsilon})_{1} \cdot \operatorname{td}_{0}\left(\tau_{X}\right) \cdot H^{d-1}\right)+\operatorname{deg}\left(\operatorname{ch}_{0}(\mathcal{\varepsilon}) \cdot \operatorname{td}_{1}\left(\tau_{X}\right) \cdot H^{d-1}\right) \\
& =\operatorname{deg}\left(c_{1}(\mathcal{\varepsilon}) \cdot H^{d-1}\right)+\frac{r k(\mathcal{\varepsilon})}{2} \operatorname{deg}\left(c_{1}\left(\tau_{X}\right) \cdot H^{d-1}\right) .
\end{aligned}
$$

In particular, for $\mathcal{\varepsilon}=\mathcal{O}_{X}$, we have

$$
\alpha_{d-1}\left(\mathcal{O}_{X}\right)=\frac{1}{2} \operatorname{deg}\left(c_{1}\left(\tau_{X}\right) \cdot H^{d-1}\right)
$$

and the first part of the lemma follows. The second part is consequence of basic properties of Chern classes.

Lemma 5.2. Let $\mathcal{\varepsilon}$ be a locally free $\mathcal{O}_{X}$-module. Then, $r k\left(\pi^{*} \mathcal{\varepsilon}\right)=r k(\mathcal{\varepsilon})$ and $\operatorname{deg}\left(\pi^{*} \mathcal{\varepsilon}\right)=|G| \operatorname{deg}(\mathcal{\varepsilon})$, in particular $\mu\left(\pi^{*} \mathcal{E}\right)=|G| \mu(\mathcal{E})$. Let $\mathcal{G}$ be a locally free $\mathcal{O}_{Y}$-module. Then, $r k\left(\pi_{*} \mathcal{G}\right)=|G| r k(\mathcal{G})$ and $\operatorname{deg}\left(\pi_{*} \mathcal{G}\right)=\operatorname{deg}(\mathcal{G})$, in particular $|G| \mu\left(\pi_{*} \mathcal{G}\right)=\mu(\mathcal{G})$.

Proof. Statements about the rank follow from the general theory of finite covers. Now, as an étale cover is affine, we have $h^{i}(Y, \mathcal{G})=h^{i}\left(X, \pi_{*} \mathcal{G}\right)$ for any coherent sheaf on $Y$; thus, $P(Y, \mathcal{G})=P\left(X, \pi_{*} \mathcal{G}\right)$ and $\alpha_{d-1}(\mathcal{G})=\alpha_{d-1}\left(\pi_{*} \mathcal{G}\right)$. On the other hand, by Lemma 5.1 we have that $\operatorname{deg}\left(\pi_{*} \mathcal{O}_{Y}\right)=c_{1}\left(\pi_{*} \mathcal{O}_{Y}\right) \cdot H^{d-1}=c_{1}\left(\pi_{*} \pi^{*} \mathcal{O}_{X}\right) \cdot H^{d-1}=\pi_{*}\left(c_{1}\left(\pi^{*} \mathcal{O}_{X}\right)\right) \cdot H^{d-1}=|G| c_{1}\left(\mathcal{O}_{X}\right)$. $H^{d-1}=0$, thus $\alpha_{d-1}\left(\pi_{*} \mathcal{O}_{Y}\right)=|G| \alpha_{d-1}\left(\mathcal{O}_{X}\right)$. In general, for a coherent sheaf $\mathcal{G}_{\text {, w }}$ we have that $\operatorname{deg}(\mathcal{G})=\alpha_{d-1}(\mathcal{G})-r k(\mathcal{G}) \alpha_{d-1}\left(\mathcal{O}_{Y}\right)=\alpha_{d-1}\left(\pi_{*} \mathcal{G}\right)-r k(\mathcal{G})|G| \alpha_{d-1}\left(\mathcal{O}_{X}\right)=\operatorname{deg}\left(\pi_{*} \mathcal{G}\right)$.

Finally, let $\mathcal{\varepsilon}$ be a locally free sheaf on $X$, then $\operatorname{deg}\left(\pi^{*} \mathcal{\varepsilon}\right)=\operatorname{deg}\left(\pi_{*} \pi^{*} \mathcal{\varepsilon}\right)=\operatorname{deg}(\mathcal{\varepsilon} \otimes$ $\left.\pi_{*} \mathcal{O}_{Y}\right)=r k\left(\pi_{*} \mathcal{O}_{Y}\right) \operatorname{deg}(\mathcal{\varepsilon})+r k(\mathcal{\varepsilon}) \operatorname{deg}\left(\pi_{*} \mathcal{O}_{Y}\right)=|G| \operatorname{deg}(\mathcal{\varepsilon})$.

Lemma 5.3. Let $\mathcal{\varepsilon}$ be a locally free $\mathcal{O}_{\mathrm{X}}$-module. Then, $\mathcal{\varepsilon}$ is $\mu$-H-semistable if and only if $\pi^{*} \mathcal{\varepsilon}$ is $\mu$-H-semistable.

Proof. If $\mathcal{\varepsilon}$ is not $\mu$-H-semistable, then there is a submodule $\mathcal{F}$ with $\mu(\mathcal{F})>\mu(\mathcal{E})$, so, by Lemma 5.2, $\mu\left(\pi^{*} \mathcal{F}\right)>\mu\left(\pi^{*} \mathcal{\varepsilon}\right)$ and $\pi^{*} \mathcal{E}$ is not $\mu$-H-semistable.

For the converse, suppose that $\pi^{*} \varepsilon$ is not $\mu-\pi^{*} H$-semistable, then, by the HarderNarasimhan filtration theorem, there exists a unique submodule $\mathcal{G}$ such that it is $\mu-\pi^{*} H$ semistable and $\mu(\mathcal{G}) \geq \mu(\mathscr{l})$ for all submodule of $\pi^{*} \mathcal{E}$; by the uniqueness, it is invariant under the action of $G$, so, by Theorem 1 in [6, page 111], there exists an $\mathcal{O}_{X}$-submodule $\mathcal{F}$ such that $\mathcal{G} \simeq \pi^{*} \mathcal{F}$ and, by previous lemma, $\mu(\mathcal{F})>E$.

Proof of Proposition 3.1. Suppose that $\pi^{*} \varepsilon$ is $\mu-H$-polystable, so by the previous lemma $\varepsilon$ is $\mu$-H-semistable and by the Jordan-Holder filtration theorem there exists a destabilizing 
submodule $\mathcal{F}$ such that it is $\mu$-H-stable and $\mu(\mathcal{F})=\mu(\mathcal{\varepsilon})$, so by previous lemmas $\pi^{*} \mathscr{F}$ is $\mu-\pi^{*} H$-semistable and $\mu\left(\pi^{*} \mathscr{F}\right)=\mu\left(\pi^{*} \mathcal{E}\right)$ and so it must be a direct summand of $\pi^{*} \mathcal{E}$. Let $\pi^{*} \mathscr{f} \stackrel{i}{\rightarrow} \pi^{*} \varepsilon \stackrel{p}{\rightarrow} \pi^{*} \mathcal{\Psi}$ be the inclusion and projection morphisms, so taking the direct image we have G-invariant morphisms $\mathcal{F} \otimes \pi_{*} \mathcal{O}_{Y} \stackrel{i_{*}}{\rightarrow} \varepsilon \otimes \pi_{*} \mathcal{O}_{Y} \stackrel{p_{*}}{\rightarrow} \mathcal{F} \otimes \pi_{*} \mathcal{O}_{Y}$ with $p_{*} \circ i_{*}=\operatorname{Id} q_{\otimes} \pi_{*} \mathcal{O}_{Y}$, but, now, $\pi_{*} \mathcal{O}_{Y}=\bigoplus_{V \in O} V \otimes \mathcal{E}_{V}$; hence, we have $\mathcal{F} \otimes V \otimes \mathcal{E}_{V} \stackrel{i_{*}}{\rightarrow} \mathcal{\varepsilon} \otimes V \otimes \mathcal{E}_{V} \stackrel{p_{*}}{\rightarrow} \mathcal{F} \otimes V \otimes \mathcal{E}_{V}$ for each irreducible representation $V$; in particular for the trivial representation $V_{0}$ we have $\mathcal{E}_{V_{0}}=\mathcal{O}_{X}$, then $\mathcal{F}$ is a direct summand of $\mathcal{\varepsilon}$.

Now, suppose that $\varepsilon$ is $\mu$-H-stable; again, by the lemma above, $\pi^{*} \varepsilon$ is $\mu$ - $\pi^{*} H$ semistable, so let $\mathcal{G}$ be a destabilizing submodulo for it. Thus, taking the sum $\sum_{g \in G} g^{*} \mathcal{G}$, we obtain a G-invariant $\mu-\pi^{*} H$-polystable submodule of $\pi^{*} \varepsilon$ which must be the pullback of a $\mu$ - $H$-polystable subsheaf $\mathcal{F}$ of $\mathcal{E}$ with the same slope, so $\mathcal{F}=\mathcal{E}$ and then $\pi^{*} \mathcal{E}=\sum_{g \in G} g^{*} \mathcal{G}$.

\section{References}

[1] M. S. Narasimhan and C. S. Seshadri, "Stable and unitary vector bundles on a compact Riemann surface," Annals of Mathematics, vol. 82, pp. 540-567, 1965.

[2] M. Lübke and A. Teleman, The Kobayashi-Hitchin Correspondence, World Scientific Publishing, River Edge, NJ, USA, 1995.

[3] D. Gieseker, "Stable vector bundles and the Frobenius morphism," Annales Scientifiques de l'École Normale Supérieure, vol. 6, pp. 95-101, 1973.

[4] A. Moriwaki, "Relative Bogomolov's inequality and the cone of positive divisors on the moduli space of stable curves," Journal of the American Mathematical Society, vol. 11, no. 3, pp. 569-600, 1998.

[5] A. Langer, "Moduli spaces of sheaves and principal G-bundles," Proceedings of Symposia in Pure Mathematics, vol. 80, pp. 273-308, 2009.

[6] D. Mumford, Abelian Varieties, Oxford University Press, London and Tata Institute of Fundamental Research, Bombay, India, 1970.

[7] C. W. Curtis and I. Reiner, Representation Theory of Finite Groups and Associative Algebras, Wiley Classics Library, John Wiley \& Sons, New York, NY, USA, 1962.

[8] D. Huybrechts and M. Lehn, The Geometry of Moduli Spaces of Sheaves, Aspects of Mathematics, E31, Friedrick Vieweg and Son, 1997.

[9] W. Fulton and J. Harris, Representation Theory: A First Course, vol. 129 of Graduate Texts in Mathematics, Springer-Verlag, New York, NY, USA, 1991.

[10] A. Tarski, A Decision Method for Elementary Algebra and Geometry, Rand Corporation Publication, 1948. 


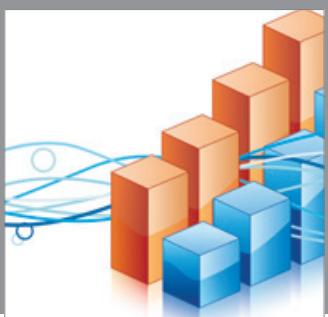

Advances in

Operations Research

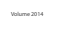

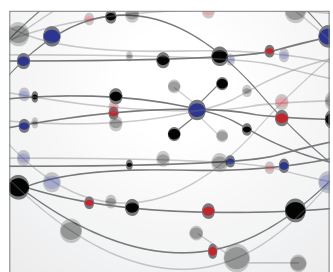

\section{The Scientific} World Journal
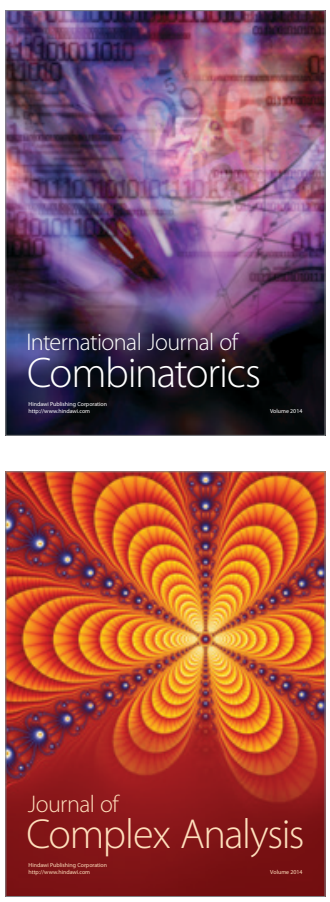

International Journal of

Mathematics and

Mathematical

Sciences
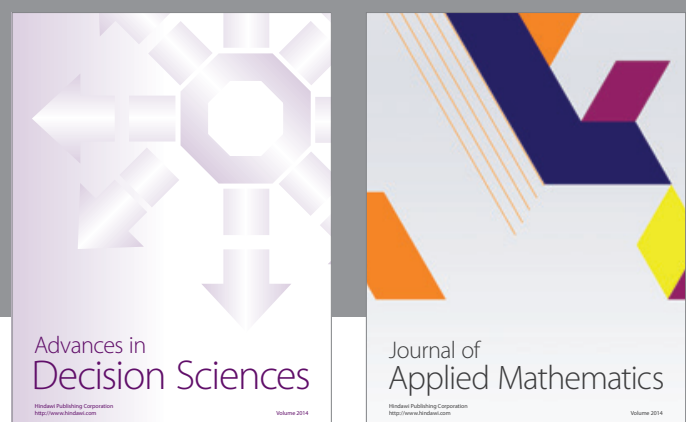

Journal of

Applied Mathematics
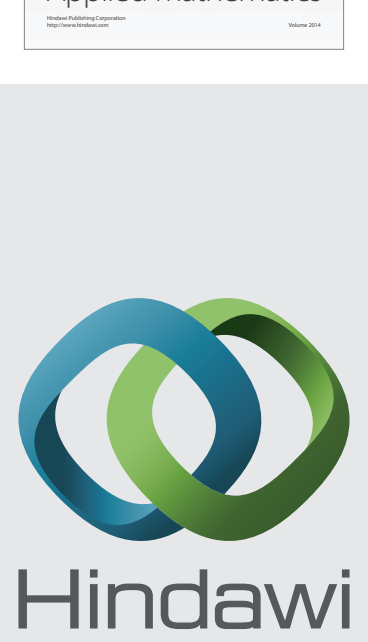

Submit your manuscripts at http://www.hindawi.com
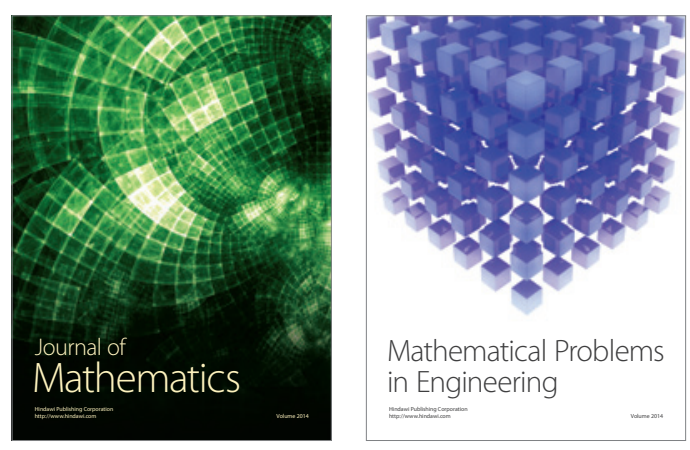

Mathematical Problems in Engineering
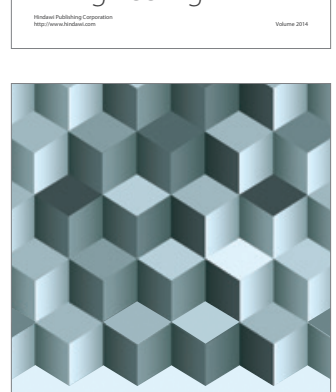

Journal of

Function Spaces
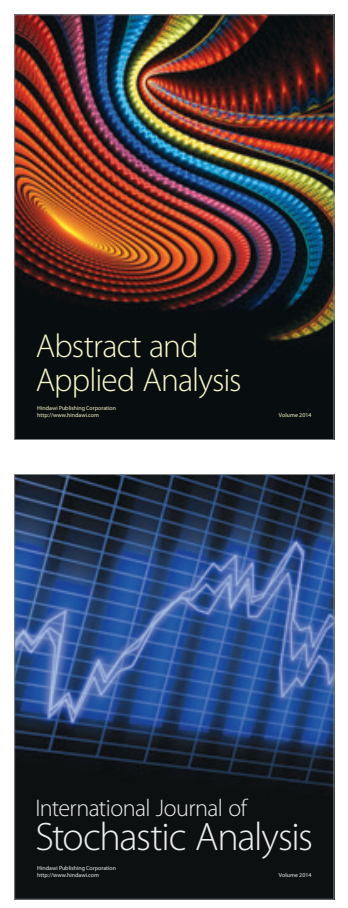

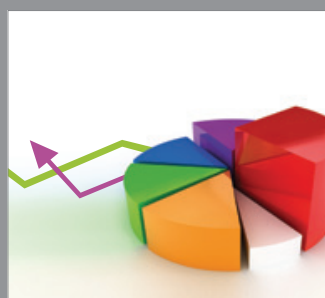

ournal of

Probability and Statistics

Promensencen
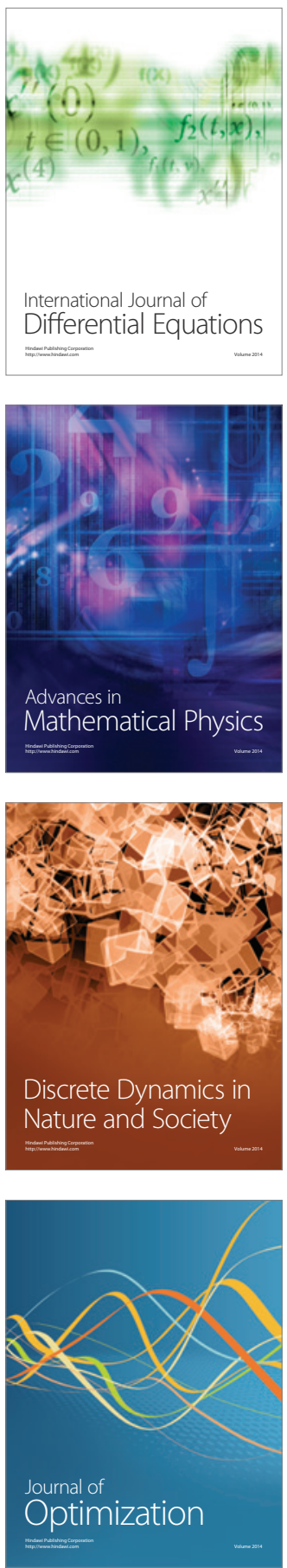